\title{
RNAseq analysis of fast skeletal muscle in restriction-fed transgenic coho salmon (Oncorhynchus kisutch): an experimental model uncoupling the growth hormone and nutritional signals regulating growth
}

\author{
Daniel Garcia de la serrana ${ }^{1 *}$, Robert H Devlin² and lan A Johnston ${ }^{1 *}$
}

\begin{abstract}
Background: Coho salmon (Oncorhynchus kisutch) transgenic for growth hormone (Gh) express Gh in multiple tissues which results in increased appetite and continuous high growth with satiation feeding. Restricting Gh-transgenics to the same lower ration (TR) as wild-type fish (WT) results in similar growth, but with the recruitment of fewer, larger diameter, muscle skeletal fibres to reach a given body size. In order to better understand the genetic mechanisms behind these different patterns of muscle growth and to investigate how the decoupling of $\mathrm{Gh}$ and nutritional signals affects gene regulation we used RNA-seq to compare the fast skeletal muscle transcriptome in TR and WT coho salmon.

Results: Illumina sequencing of individually barcoded libraries from 6 WT and 6 TR coho salmon yielded 704,550,985 paired end reads which were used to construct 323,115 contigs containing 19,093 unique genes of which >10,000 contained $>90 \%$ of the coding sequence. Transcripts coding for 31 genes required for myoblast fusion were identified with 22 significantly downregulated in TR relative to WT fish, including 10 (vaspa, cdh15, graf1, crk, crkl, dock1, trio, plekho 1a, cdc42a and dock5) associated with signaling through the cell surface protein cadherin. Nineteen out of 44 (43\%) translation initiation factors and 14 of 47 (30 \%) protein chaperones were upregulated in TR relative to WT fish.

Conclusions: TR coho salmon showed increased growth hormone transcripts and gene expression associated with protein synthesis and folding than WT fish even though net rates of protein accretion were similar. The uncoupling of Gh and amino acid signals likely results in additional costs of transcription associated with protein turnover in TR fish. The predicted reduction in the ionic costs of homeostasis in TR fish associated with increased fibre size were shown to involve multiple pathways regulating myotube fusion, particularly cadherin signaling.
\end{abstract}

Keywords: Muscle growth, teleost fish, Growth hormone transgenics, Skeletal muscle transcriptome, Fish nutrition

\section{Background}

The growth hormone (Gh) axis in coho salmon (Oncorhynchus kisutch) and other teleosts is normally tightly coupled to energy intake and is modulated by a large number of environmental factors [1]. The normal feedback control systems are essentially disabled in growth hormone-transgenics due to the extra-pituitary

\footnotetext{
* Correspondence: dgdlsc@st-andrews.ac.uk; iaj@st-andrews.ac.uk

${ }^{1}$ Scottish Oceans Institute, School of Biology, University of St Andrews, KY16 8 LB St Andrews, Scotland, UK

Full list of author information is available at the end of the article
}

expression of Gh in other tissues [2, 3]. High constitutive expression of $G$ h results in increased aggressiveness, appetite and foraging activity $[4,5]$ leading to continuous fast growth when fish are fed to satiation [6]. Gh stimulates insulin-like growth factor-1 (Igf1) secretion in the liver. Circulating Igfs bind to Igf receptors and activate the signalling cascades that regulate protein synthesis. Plasma Igf1 and amino acids from the diet further stimulate Igf synthesis in muscle and other tissues via paracrine action $[7,8]$. Gh-transgenics exhibit increased plasma insulin-like growth factor-1 (Igf1; 25 ng/ml), 
tissue igf1 mRNA and Gh-receptor ( $g h r$ ) levels relative to wild-type fish (circulating Igf1 was $\sim 8 \mathrm{ng} / \mathrm{ml}$ in WT) [3]. Feeding coho salmon Gh-transgenics below appetite (restriction-fed transgenics, TR) with a similar ration size as wild-type fish (WT) results in an uncoupling of the growth hormone and nutritional signals regulating growth. TR fish grow more slowly and show lower circulating Igf1 than Gh-transgenic fish fed to satiation (TF) $(10 \mathrm{ng} / \mathrm{ml}$ in TR compared with $25 \mathrm{ng} / \mathrm{ml}$ to TF animals), which indicates the nutritional regulation of igfl expression remains functional at the target tissue level [3]. TR animals showed higher circulating Gh $(8 \mathrm{ng} / \mathrm{ml})$ levels and unchanged $g h r$ mRNA relative to Gh-transgenics fed to satiation (TF) ( $4 \mathrm{ng} / \mathrm{ml}$ circulating $\mathrm{Gh}$ ), but retained higher feeding motivation and foraging activity than WT [3].

Fast skeletal muscle fibres are formed in teleost fish immediately following somite formation and then in the juvenile/adult stages by processes of stratified and mosaic hyperplasia $(\mathrm{MH})[9,10]$. Myoblast-myoblast fusion results in the formation of a nascent myotube which elongates and gains additional nuclei through myoblast-myotube fusion. Activation of terminal differentiation and sarcomere formation then results in the production of an immature muscle fibre. Typically, myotube production in fast muscle continues until the fish reaches around 40-50 \% of their maximum attainable adult body length [11].

Hill et al. [12] provided indirect evidence that Ghtransgenic coho salmon fed to satiation showed enhanced muscle growth by hyperplasia. In a recent study we tested this hypothesis by measuring the number and size distribution of fast myotomal muscle fibres in three groups of coho salmon of similar body length: 1-year old Gh-transgenics fed to satiation (TF), 2-year old restrictionfed Gh-transgenics (TR) and wild type (WT) [13]. TF coho salmon recruited fast muscle fibres at twice the rate as WT fish, but showed a similar contribution of hyperplasia and hypertrophy to reach a given body length i.e. the hypothesis of an increased importance of hyperplasia in transgenics was not supported. Unexpectedly, TR recruited $49 \%$ fewer fibres with a $20 \%$ higher fibre diameter than either WT or TF fish and had larger diameter fibres across the whole range of fibre sizes, i.e. increased hypertrophy was evident for all cohorts of fibres produced during ontogeny [13]. There is direct experimental evidence from inhibitor and Nuclear Magnetic Resonance (NMR) studies that larger diameter muscle fibres have lower costs of ionic homeostasis than smaller ones due to their lower surface to volume $(\mathrm{S} / \mathrm{V})$ ratio $[14,15]$. Thus the $\sim 40 \%$ reduction in fibre $\mathrm{S} / \mathrm{V}$ ratio in TR relative to WT fish would be expected to produce proportional savings in routine maintenance costs [13]. Previously we suggested that adjustments in fibre size might permit the reallocation of energy from maintenance to locomotion which would help explain why calorie- restricted transgenics grow at the same rate as WT fish whilst exhibiting markedly higher activity levels [13].

Preliminary, studies indicated that several genes associated with myotube formation were downregulated in TR relative to WT fish [13]. In order to gain a broader understanding of fast skeletal muscle gene regulation in TR compare to WT fish we have now carried out RNA-seq in 6 TR and $6 \mathrm{WT}$ individual coho salmon for which we had associated data on the number and size of fast muscle fibres [13], and validated the results by qPCR analysis. RNA was sequenced using Illumina HiSeq2000 and mapped reads (DESEQ normalized counts) were used to study global differences in digital gene expression (DGE) between groups. Following enriched Gene Ontology (GO) analysis we tested specific hypotheses about the effects of uncoupling growth hormone and nutrient signals by analysing the expression of genes related to growth hormone signalling, protein translation, protein-folding and myoblast fusion.

\section{Results and discussion \\ Transcriptome analysis}

Total RNA from TR and WT fish ( $n=6$ per condition) with known differences in muscle cellularity [13] were sequenced in 2 lines of Illumina Hiseq2000 . A total of $704,550,985$ paired-end reads were de novo assembled into 323,115 contigs with average length $>1,000 \mathrm{bp}$ and $\mathrm{N}_{50}>2,000$ bp (Table 1; Additional file 1). Using BLASTx against the NCBI non-redundant (nr) database we successfully identified a total of 153,839 contigs and, from them, a total of 149,738 were also annotated with Gene Ontology (GO) terms (Table 1). A total of 19,093 unique genes were identified in the fully annotated transcriptome (Additional file 1). The teleost linage underwent a whole-genome duplication (WGD) relative to the last common ancestor with humans around 350320 Mya [16], increasing gene content by $15-20 \%$ [17]. The salmonids experienced a fourth lineage-specific WGD (4R) around 88Mya, and approximately $50 \%$ of the resulting paralogues are still present in extant species [18]. Several examples of gene expansion due to the salmonid WGD have been described in detail [19, 20]. In order to estimate the representation of $4 R$ paralogues in the transcriptome 38 well-characterized Atlantic salmon (Salmo salar) paralogues gene families were blasted (tBLASTn) against the coho salmon de novo transcriptome. Seventeen out of 38 paralogues were unequivocally identified $(44 \%)$ and three more appeared with very low e-values (Table 2), though not all can be expected to be expressed in skeletal muscle. 21,995 protein-coding genes have been reported for the human genome of which 12,557 are expressed in the muscle transcriptome [21]. The $51 \%$ higher gene content of the coho skeletal muscle transcriptome $(19,093)$ than in humans likely reflects this history of 
Table 1 Sequencing and de novo Trinity assembly metrics

\begin{tabular}{lll}
\hline & WT & TR \\
\hline Paired-end reads & $351,369,759$ & $353,181,226$ \\
Reads assembly & $267,224,210$ & $254,344,286$ \\
Percentage assembly & 71.53 & 72.04 \\
Contigs & 323,115 & \\
Average length (bp) & 1,103 & \\
N50 (bp) & 2,142 & \\
Components annotated & 149,738 & \\
Unigenes & 19,093 & \\
Complete CDS & 10,030 & \\
KAAS annotated maps & 309 &
\end{tabular}

TR: restriction-fed growth hormone-transgenic coho salmon; WT: wild-type coho salmon; N50: average length that contains at least half of the sum of the lengths for all contigs; bp: base pair; CDS: coding sequence; KAAS: KEEG automatic annotation service

the succesive WGD. Furthermore, the continuous expression of Gh might also increase the number of genes expressed at detectable levels in the muscle.

Annotated contigs were blasted against the KEGG database using the online web-server KAAS to map them against the main metabolic and signaling pathways, yielding a total of 309 KEGG maps where coho salmon contigs were present (Table 1; Hierarchical file with all the details of the KEGG mapped contigs can be found in Additional file 1). Comparative analysis between annotated contigs against the annotated zebrafish proteome (Danio rerio) allowed us to identify 10,030 genes estimated to contain over $90 \%$ of the predicted coding sequence (CDS) (Additional file 2). We also found that 1000 contigs were over $>100 \%$ of the CDS, indicating that some coho salmon genes were between 1-20\% longer than their zebrafish orthologues (Additional file 2).

\section{Digital Gene Expression (DGE)}

Paired-end reads from individual fish libraries were mapped against the de novo transcriptome (Additional file 3). Mapped reads were normalized by contig length and library depth following a negative binomial using DESEQ and used for DGE analysis. Global DGE comparison between TR and WT yielded an initial list of 384 contigs that were differentially expressed (FDR $<0.05)$. Contigs with $<15$ normalized reads mapped and a fold-change $<2 \log _{2}$ were discarded. Redundant contigs for all genes from the initial global DGE list were identified in the annotated transcriptome and their DESEQ-counts values were individually investigated. Those genes for which all-redundant "sister" contigs were found to show consistent, but not necessarily significant, changes in expression between groups were retained (see Methods). Care was taken to identify $4 R$ and $3 R$-paralogues that might be annotated with the same ID by exploring the alignment of the sister contigs. After curation for redundancy and quality control, a total of 186 genes from TR and 199 from WT were considered to be differentially expressed (Additional file 4).

RNAseq has many advantages, but it does present challenges with respect to statistical analysis and interpretation. Firstly, transcription is not tightly coupled to translation in eukaryotes, complicating inferences about the functional significance of changes in transcript abundance. Secondly, the activity of signalling pathways is often dependent on posttranslational modifications such as phosphorylation and/or changes in compartmentalisation within the cell. In addition, functional interpretations based on transcript abundance cannot distinguish between mRNA transcription vs degradation rates. It is also easy to fall into the trap of providing a series of post hoc stories when reporting transcriptomic data rather than testing a priori hypotheses. Standard corrections for multiple testing coupled with long gene lists also leads to a very high barrier for establishing statistical significance, leading to type- 2 statistical errors.

We therefore used a multistep process to analyse the DGE results from TR and WT fish fed the same ration. Firstly, enriched Gene Ontology (GO) analysis was used to identify initial categories of those genes obtained from the global analysis for further investigation (Additional file 4). For the TR fish, GO analysis revealed an enrichment in genes related to "macromolecule metabolic process" (GO:44260), "RNA metabolic process" (GO:16070), "regulation of translation" (GO:6417), "translation factor activity, nucleic acid binding" (GO:8135) or "RNA binding" (GO:3723) (FDR < 0.05; Table 3). WT animals had a higher expression of genes related to "autophagy" (GO:6914), "cellular metabolic process" (GO:44237), “catabolic process" (GO:9056) and "vacuole organization” (GO:7033) (FDR < 0.05; Table 3). Based on GO enrichment analysis and previous works [13] we decided to specifically test the expression of genes related to myoblast fusion, protein synthesis, protein degradation by the proteasome system, protein folding and also the Gh-Igf receptors and ligands. KAAS annotation results and a literature survey of the results of functional analyses were used to identify the genes to include. 363 genes were identified in total (Additional file 4), including $4 \mathrm{R}$ and $3 \mathrm{R}$ paralogues where identified (examples of $4 \mathrm{R}$ salmonid paralogues are in Fig. 1; trees for the potential $4 R$ paralogues identified can be found in Additional file 5). DESEQ-counts (considered more stringent and a better estimation of gene expression than FPKM) from individual animals for each gene were then retrieved in order to test specific a priori hypotheses. 
Table 2 Estimation of paralogues content in the coho salmon transcriptome

\begin{tabular}{|c|c|c|c|c|}
\hline S. salar paralogue & Coho salmon contig & E-value & Id (\%) & Alignment (AA) \\
\hline igfbplal & comp120142_c0_seq3 & 0 & 95 & 807 \\
\hline igfbpla2 & $\mathrm{n} / \mathrm{a}$ & $\mathrm{n} / \mathrm{a}$ & $\mathrm{n} / \mathrm{a}$ & $\mathrm{n} / \mathrm{a}$ \\
\hline igfbp $1 b 1$ & $\mathrm{n} / \mathrm{a}$ & $\mathrm{n} / \mathrm{a}$ & $\mathrm{n} / \mathrm{a}$ & $\mathrm{n} / \mathrm{a}$ \\
\hline igfbp $1 b 2$ & $\mathrm{n} / \mathrm{a}$ & $\mathrm{n} / \mathrm{a}$ & $\mathrm{n} / \mathrm{a}$ & $\mathrm{n} / \mathrm{a}$ \\
\hline igfbp $2 a$ & $\mathrm{n} / \mathrm{a}$ & $\mathrm{n} / \mathrm{a}$ & $\mathrm{n} / \mathrm{a}$ & $\mathrm{n} / \mathrm{a}$ \\
\hline igfbp $2 b 1$ & $\mathrm{n} / \mathrm{a}$ & $\mathrm{n} / \mathrm{a}$ & $\mathrm{n} / \mathrm{a}$ & $\mathrm{n} / \mathrm{a}$ \\
\hline igfbp $2 b 2$ & comp132077_c2_seq30 & 0.24 & 100 & 20 \\
\hline igfbp3al & comp125786_c0_seq1 & 0 & 96 & 889 \\
\hline igfbp $3 a 2$ & $\mathrm{n} / \mathrm{a}$ & $n / a$ & $\mathrm{n} / \mathrm{a}$ & $\mathrm{n} / \mathrm{a}$ \\
\hline igfbp $3 b 1$ & $\mathrm{n} / \mathrm{a}$ & $\mathrm{n} / \mathrm{a}$ & $\mathrm{n} / \mathrm{a}$ & $\mathrm{n} / \mathrm{a}$ \\
\hline igfbp $3 b 2$ & comp712690_c0_seq1 & $1.00 \mathrm{E}-154$ & 96 & 324 \\
\hline igfbp4 & $\mathrm{n} / \mathrm{a}$ & $\mathrm{n} / \mathrm{a}$ & $\mathrm{n} / \mathrm{a}$ & $\mathrm{n} / \mathrm{a}$ \\
\hline igfbp $5 a$ & $\mathrm{n} / \mathrm{a}$ & $\mathrm{n} / \mathrm{a}$ & $\mathrm{n} / \mathrm{a}$ & $\mathrm{n} / \mathrm{a}$ \\
\hline igfbp5b1 & comp126977_c0_seq9 & 0 & 96 & 807 \\
\hline igfbp $5 b 2$ & $\mathrm{n} / \mathrm{a}$ & $\mathrm{n} / \mathrm{a}$ & $\mathrm{n} / \mathrm{a}$ & $\mathrm{n} / \mathrm{a}$ \\
\hline igfbp6a1 & $\mathrm{n} / \mathrm{a}$ & $\mathrm{n} / \mathrm{a}$ & $\mathrm{n} / \mathrm{a}$ & $\mathrm{n} / \mathrm{a}$ \\
\hline igfbp6a2 & $\mathrm{n} / \mathrm{a}$ & $\mathrm{n} / \mathrm{a}$ & $\mathrm{n} / \mathrm{a}$ & $\mathrm{n} / \mathrm{a}$ \\
\hline igfbp6b1 & $\mathrm{n} / \mathrm{a}$ & $\mathrm{n} / \mathrm{a}$ & $\mathrm{n} / \mathrm{a}$ & $\mathrm{n} / \mathrm{a}$ \\
\hline igfbp $6 b 2$ & comp130388_c0_seq9 & 0 & 98 & 609 \\
\hline akirin 1(1a) & comp126876_c0_seq6 & 0 & 96 & 638 \\
\hline akirin 1(1b) & comp126876_c0_seq2 & 0 & 96 & 655 \\
\hline akirin 1(2a) & comp128245_c1_seq9 & 0 & 95 & 690 \\
\hline akirin $1(2 b)$ & comp128245_c1_seq10 & 0 & 98 & 592 \\
\hline akirin 2(1a) & $\mathrm{n} / \mathrm{a}$ & $\mathrm{n} / \mathrm{a}$ & $\mathrm{n} / \mathrm{a}$ & $\mathrm{n} / \mathrm{a}$ \\
\hline akirin 2(1b) & comp127034_c0_seq3 & 0 & 98 & 472 \\
\hline akirin 2(2a) & $\mathrm{n} / \mathrm{a}$ & $\mathrm{n} / \mathrm{a}$ & n/a & $\mathrm{n} / \mathrm{a}$ \\
\hline akirin 2(2b) & comp126525_c1_seq7 & 0 & 98 & 546 \\
\hline hsp90aa1.3 & comp132154_c0_seq29 & 0 & 92 & 1386 \\
\hline hsp90aa1.4 & comp132154_c0_seq71 & 0 & 95 & 1281 \\
\hline hsp90aa1.1 & $\mathrm{n} / \mathrm{a}$ & $\mathrm{n} / \mathrm{a}$ & $\mathrm{n} / \mathrm{a}$ & $\mathrm{n} / \mathrm{a}$ \\
\hline hsp90aa1.2 & $\mathrm{n} / \mathrm{a}$ & $\mathrm{n} / \mathrm{a}$ & $\mathrm{n} / \mathrm{a}$ & $\mathrm{n} / \mathrm{a}$ \\
\hline hsp90b1 & comp122350_c0_seq5 & 0 & 97 & 619 \\
\hline hsp90b2 & $\mathrm{n} / \mathrm{a}$ & $\mathrm{n} / \mathrm{a}$ & n/a & $\mathrm{n} / \mathrm{a}$ \\
\hline aqp8 & comp132382_c1_seq19 & $1.00 \mathrm{E}-28$ & 95 & 82 \\
\hline aqp8aal & comp104094_c0_seq1 & 0 & 91 & 796 \\
\hline$a q p 8 a b$ & $\mathrm{n} / \mathrm{a}$ & $\mathrm{n} / \mathrm{a}$ & $\mathrm{n} / \mathrm{a}$ & $\mathrm{n} / \mathrm{a}$ \\
\hline aqp $8 b$ & comp450295_c0_seq1 & 0.87 & 100 & 19 \\
\hline myod1 & comp122414_c0_seq4 & 0 & 96 & 986 \\
\hline myod2 & $\mathrm{n} / \mathrm{a}$ & $n / a$ & $\mathrm{n} / \mathrm{a}$ & $n / a$ \\
\hline myolc & comp131066_c1_seq17 & 0 & 98 & 859 \\
\hline
\end{tabular}

Id: percentage of identity at amino acid level between S.salar and O.kitsuch orthologues

Alignment: Total number of amino acids successfully aligned during the tBLASTn between S.salar and O.kitsuch orthologues $\mathrm{n} / \mathrm{a}$ : Paralogues not identified in the transcriptome 
Table 3 Gene ontology analysis

\begin{tabular}{|c|c|c|c|c|}
\hline Condition & GO term & GO:ID & Number of genes & FDR \\
\hline \multicolumn{5}{|l|}{ TR } \\
\hline \multirow[t]{6}{*}{ Biological process } & Ribonucleoprotein complex biogenesis & 0022613 & 18 & $7.1 e^{-8}$ \\
\hline & RNA processing & 0006396 & 21 & $6.0 e^{-5}$ \\
\hline & Regulation of translational initiation & 0006446 & 8 & $1.7 e^{-4}$ \\
\hline & Cellular macromolecule metabolic process & 0044260 & 70 & $2.0 e^{-4}$ \\
\hline & Cellular nitrogen compounds metabolic process & 0034641 & 64 & $3.7 e^{-4}$ \\
\hline & Primary metabolic process & 0044238 & 84 & $3.7 e^{-4}$ \\
\hline \multirow[t]{5}{*}{ Molecular function } & RNA binding & 0003723 & 54 & $4.3 e^{-20}$ \\
\hline & Nucleic acid binding & 0003676 & 58 & $1.4 e^{-6}$ \\
\hline & Protein binding & 0005515 & 89 & $2.9 e^{-3}$ \\
\hline & Organic cyclic compound binding & 0097159 & 64 & $7.4 e^{-3}$ \\
\hline & Catalytic activity & 0003824 & 49 & 0.05 \\
\hline \multirow[t]{4}{*}{ Cellular component } & Ribonucleoprotein complex & 0030529 & 22 & $3.8 e^{-7}$ \\
\hline & Nuclei & 0005634 & 82 & $1.9 e^{-6}$ \\
\hline & Nucleolus & 0005730 & 22 & $2.5 e^{-5}$ \\
\hline & Intracellular organelle & 0044446 & 108 & $2.5 e^{-5}$ \\
\hline \multicolumn{5}{|l|}{ WT } \\
\hline \multirow[t]{6}{*}{ Biological process } & Macroautophagy & 0016236 & 7 & $1.5 e^{-4}$ \\
\hline & Cellular protein modification process & 0006464 & 37 & $5.5 e^{-4}$ \\
\hline & Autophagy & 0006914 & 9 & $5.5 e^{-4}$ \\
\hline & Macromolecule modification & 0043412 & 36 & $3.4 e^{-3}$ \\
\hline & Cellular response to nutrient levels & 0031669 & 8 & $4.5 e^{-3}$ \\
\hline & Protein modification by small protein conjugation & 0032446 & 16 & $4.8 e^{-3}$ \\
\hline \multirow[t]{2}{*}{ Molecular function } & Protein binding & 0005515 & 97 & $1.1 e^{-3}$ \\
\hline & Catalytic activity & 0003824 & 54 & $2.8 e^{-2}$ \\
\hline \multirow[t]{4}{*}{ Cellular component } & Vacuole & 0005773 & 21 & $1.0 e^{-6}$ \\
\hline & Cytoplasm & 0005737 & 112 & $1.0 e^{-6}$ \\
\hline & Lysosome & 0005764 & 17 & $5.0 e^{-5}$ \\
\hline & Intracellular component & 0044424 & 121 & $6.1 e^{-5}$ \\
\hline
\end{tabular}

Gene Ontology analysis from genes with significant differences in expression between restriction-fed growth hormone-transgenic coho salmon (TR) and wild-type coho salmon (WT); GO: Gene Ontology; FDR: False discovery rate

Before analysing in detail the differences in DGE between genes a Principal Components Analysis (PCA) was carried to determine if the categories selected were informative of the differences between TR and WT individuals (Fig. 2a). Components 1 and 2 generated from PCA analysis explained $61.5 \%$ of the variability of the data and were able to distinguish TR and WT in two separated clusters (Fig. 2a). This is an indication that the data used was informative with respect to the differences between groups, which is highly apparent in the hierarchical clustering shown in Fig. 2b.

\section{Growth hormone system and protein synthesis}

Nineteen genes associated with the growth hormone system ( $g h$, igf, their receptors and binding proteins) were identified in the transcriptome and filtered for redundancy as previously described. In order to capture all the biological relevant changes, the requirement that differences should be higher than $2 \log _{2}$-fold change was relaxed but not the requirement that average DESEQ-counts should be $>15$. As expected due to the transgenic nature of the coho salmon, $g h 1$ gene transcripts were 4.5 -fold higher in TR compared to WT [3] (Fig. 3). Similarly, previous studies have also reported an increase in growth hormone receptor $(g h r)$ expression in muscle [3]. However, that study didn't distinguish between ghr paralogues whereas we identified ghra.2 and ghrb.1, two of the potential 4 salmonid paralogues for the receptor (Figs. 1 and 3; Additional file 6). We found that ghra.2 was 1.4-fold higher in TR than WT fish (Fig. 3b) whereas ghrb.1 showed unaltered expression, 


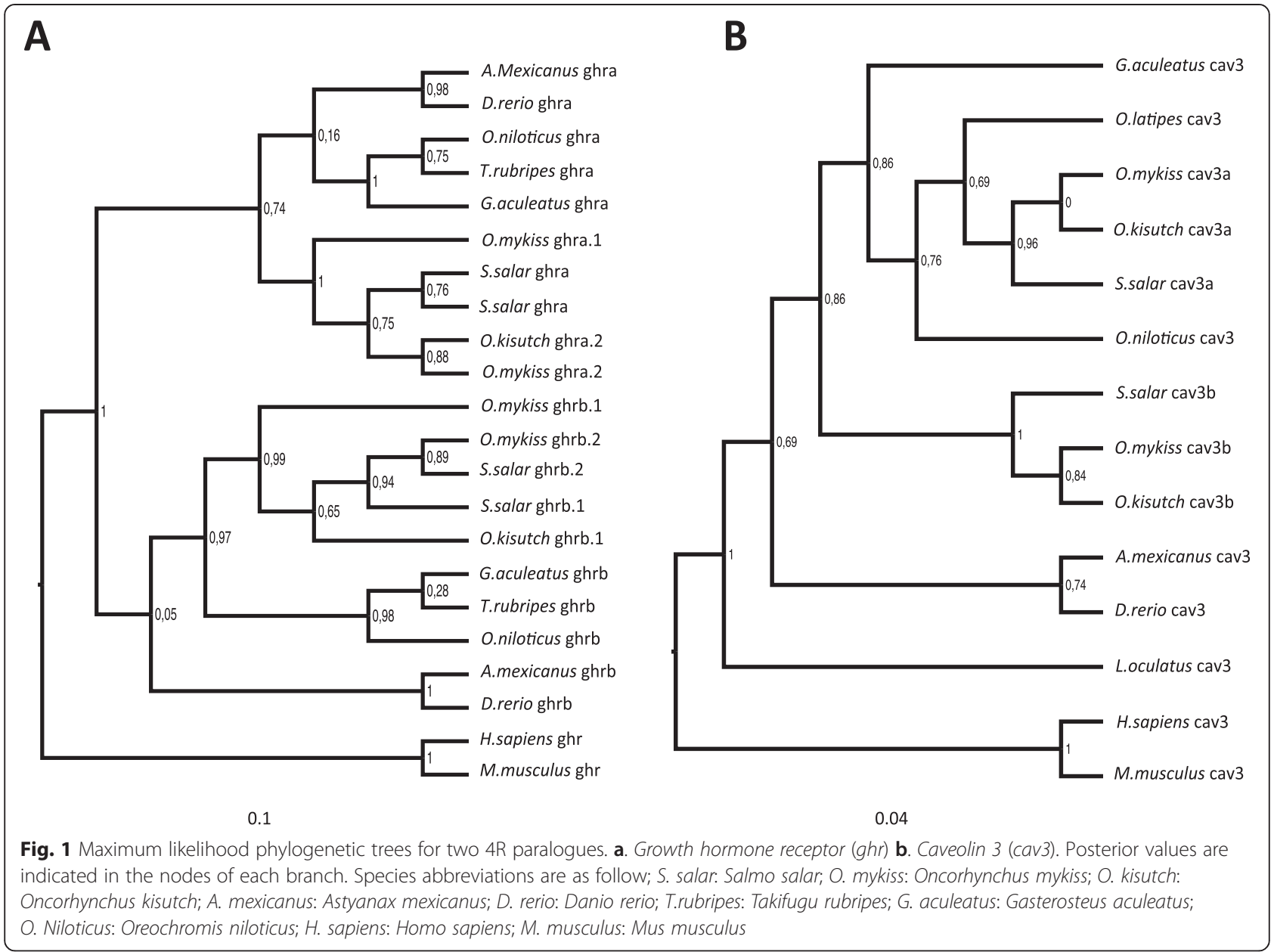

indicating differential regulation of paralogues. Igf $2 /$ igflr and the insulin receptor (inr) were downregulated 2- and 3 -fold respectively (FDR $<0.01$ ) in TR than WT fish, most likely as part of a negative feedback response to increased growth hormone expression (Fig. 3; Additional file 6).

The mTorc1 complex integrates cues from growth factors to activate ribosome biogenesis and protein synthesis via phosphorylation of ribosomal protein S6 kinase 1 [22]. mTorc1-mediated phosphorylation of the eukaryotic initiation factor $4 \mathrm{E}$ (Eif4e) binding protein (4ebp1-3) induces its dissociation from eIF4E and promotes cap-dependent translation [23]. mTorc1 is also an important part of the amino acid sensing machinery in the cell comprising Rags, Ragulator complex and v-Atpase [24]. The amino acid-Rag axis activates mTORC1 through a mechanism involving its translocation to the lysosomal surface [25]. Thus in the absence of sufficient levels of amino acids, the incorrect positioning of $\mathrm{mTorc} 1$ renders it insensitive to activation by growth factors [26]. The present transcriptome data provides strong evidence that elevated Gh levels resulted in a widespread increase in translation initiation factor mRNA with 19 out of 44 (43\%) genes significantly higher expressed in TR than WT fish, including eif4e1 and eif4e2 (Fig. 4; Additional files 6 and 7 ). It is clear from the hierarchical cluster of all 44 genes that $70 \%$ of the genes seem to have a higher expression in TR, even if not significant, than WT with only a handful of contigs more expressed in this condition, such as eif1 (Fig. 4; Additional files 6 and 7). In addition, 14 out of 47 protein chaperones genes $(30 \%)$ with roles in protein folding were significantly elevated in TR relative to WT fish, including many with known roles in muscle differentiation and sarcomere assembly such as unc45 and hsp90aa1 that are also regulated by nutrition (Fig. 5; Additional file 8) $[8,27]$. The majority of E3-ubiquitin ligases analysed, enzymes involved in protein degradation through the Proteasome system, were more highly expressed in WT than TR (however, due to individual variability in WT fish only some of the differences found were significant) (Fig. 6; Additional file 6), which indicates that TR fish were not starving. The differences in translation initiation factors, protein chaperones and E3-ubiquitin ligases expression in TR fish 


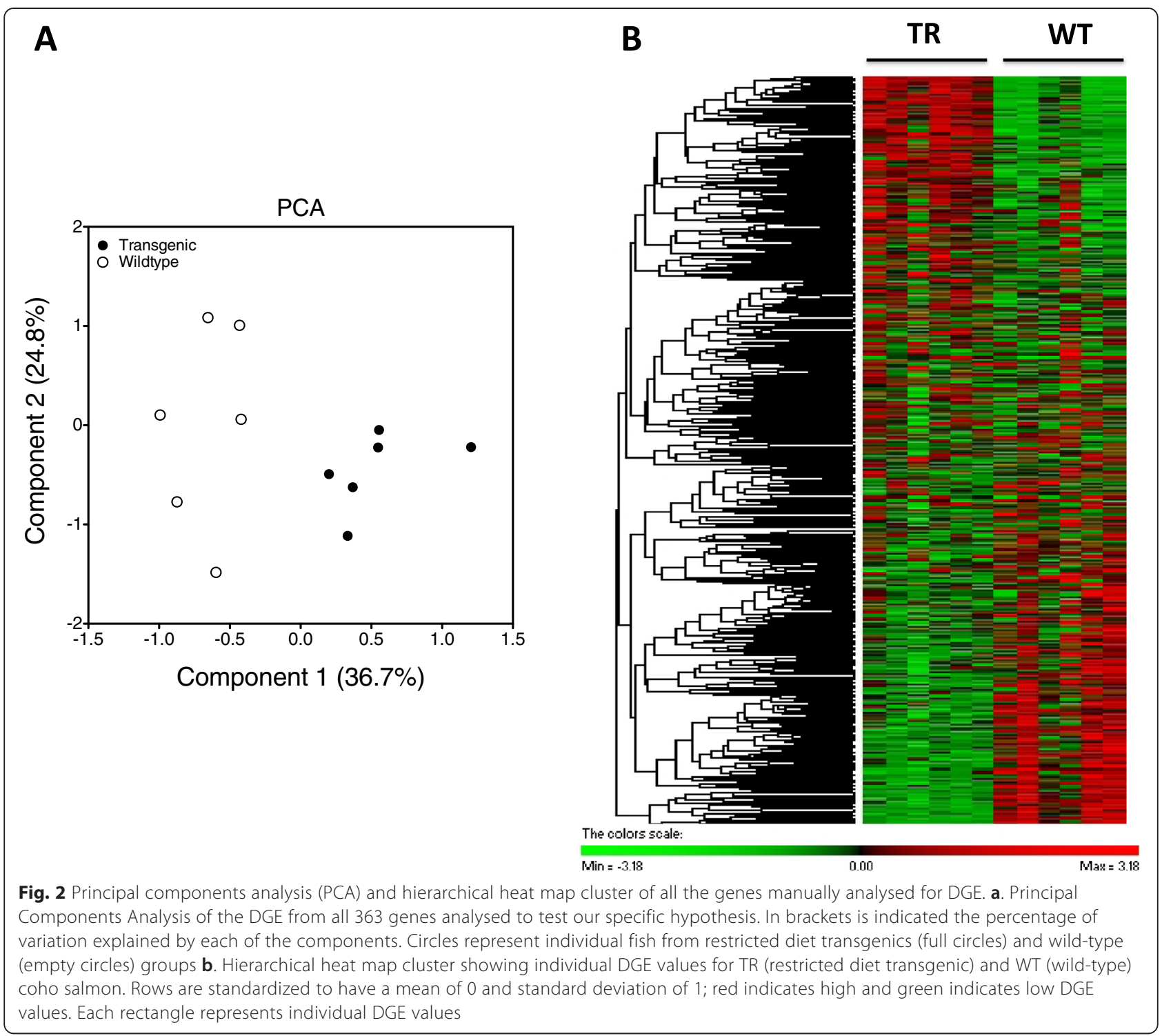

were not matched by a net increased protein accretion since TR and WT fish grew at the same rate [13], implying some increase in the metabolic costs of transcription relative to WT.

\section{Myoblast fusion}

Myoblast fusion involves multiple sarcolemmal receptors, cell-surface proteins and intracellular signaling pathways leading to membrane and actin cytoskeleton remodelling [28]. Evidence from in vivo and in vitro studies involving mouse and zebrafish models was used to construct a list of genes required for myoblast fusion and progression. A total of 99 genes including 4R-paralogues (cav3a, cav3b, cdc42a, $c d c 42 b, c d c 37 a, c d c 37 b)$ involved in myoblast fusion and differentiation were identified in the transcriptome (Additional file 6). 31 out of the 99 genes (31\%) from the list were differentially expressed between TR and WT groups of coho salmon (Fig. 7; Additional file 9) (FDR < 0.05). A total of 22 of the genes were significantly down regulated in ration-restricted transgenic fish concomitant with a reduction in the number of fast muscle fibres per myotomal cross-section [13]. The majority (10) of these genes (vaspa, cdh15, graf1, crkl, crk, dock1, trio, plekhola, $c d c 42 a$ and $d o c k 5$ ) were associated with signaling through the cell surface protein cadherin (Fig. 7a).

Morpholino antisense-oligonucleotide-mediated knockdown studies have shown that both dock 1 and dock 5 and their adaptor proteins $c r k$, crk-like ( $c r k l$ ) and the pleckstrin homology domain containing family member 1a (plekhola or ckip1) are required for the fusion of fast-type myoblasts in zebrafish [29]. Members of the Rho family of guanosine triphosphatases (GTPases), including Rac1, operate downstream of Dock1 to stimulate myoblast fusion [28]. Rac1 is also activated by M-cadherin-dependent adhesion through 


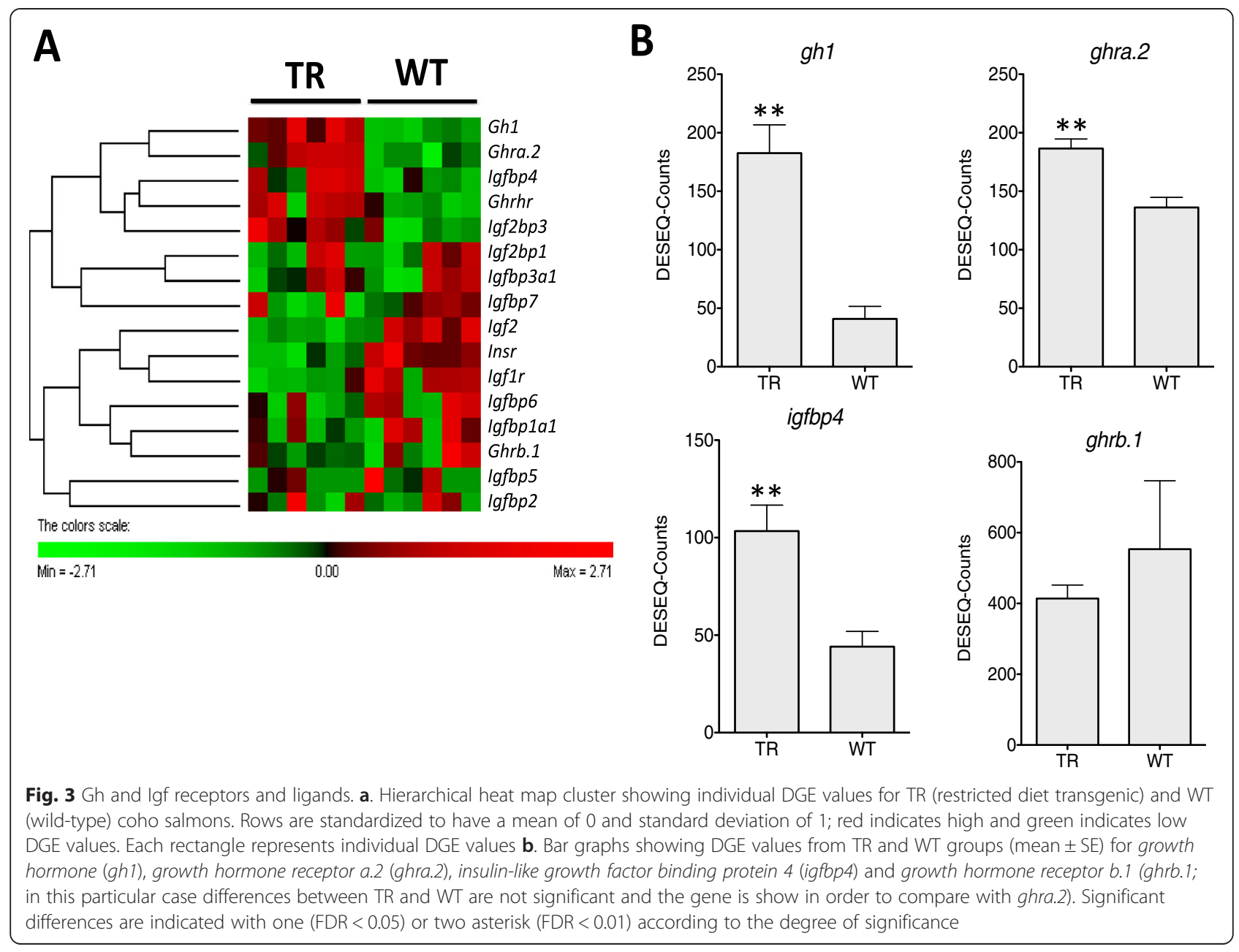

Trio during $\mathrm{C} 2 \mathrm{C} 12$ myoblast fusion [30]. M-cadherin (also known as cadherin15) and trio transcripts were also both significantly downregulated in TR relative to WT salmon (Fig. 7; Additional file 9). On the basis of differences in the defects observed in dock $^{-/-}$and trio $^{-/-}$mice it has been suggested that Trio is required for myoblast-myoblast fusion, but not for myoblast-myotube fusion [28]. A further three of the genes downregulated in TR fish were associated with integrins and focal adhesion kinase signaling (Fig. 7; Additional file 9). Also down regulated in TR relative to WT fish were $s p 1$, a component of MapkErk5 signaling pathway, $n f k b 1$ which is involved in the non-canonical NF-kB signaling and tmem $8 c$ or myomaker which is required for myoblast-myotube fusion (Fig. 7; Additional file 9) [31]. Nine (aox1, gsk-3ß, cpn3, arf6, arpc4, shp2, cfl2, mrf4 and nfatc2) out of the 99 genes on the list were significantly upregulated in TR relative to WT fish (FDR <0.05) (Fig. 7; Additional file 9). Integrin signaling is mediated by the non-receptor protein kinase Fak promoting myoblast fusion and an associated increase in Caveolin-3 [32], transcripts of which were downregulated in TR relative to WT fish (Fig. 7; Additional file 9). The differences in DGE observed are consistent with a higher myoblast fusion activity in WT when compared with TR, consistent with the observed differences in fibre number [13].

\section{Quantitative PCR data validation of DGE}

In order to validate the results obtained from DGE we analysed the expression of 23 genes covering different ranges of expression (including $3 \mathrm{R}$ and $4 \mathrm{R}$-paralogues cav3a, cav3b, cdc42a, cdc42b, vaspa, vaspb, ghra.2 and ghrb.1) by quantitative PCR (qPCR). The qPCR data supports the DGE estimations reported with similar differences between treatments for the individual genes (Additional file 10). Correlation analysis was used to examine the relationship between the levels of expression for individual genes, that were found to be equivalent irrespective of whether the data were expressed as DESEQ-counts and average $C t$ values $\left(R^{2}=0.53 ; P<0.0001\right)$ or DGE and $\mathrm{qPCR}$ fold-change $\left(\mathrm{R}^{2}=0.71 ; P<0.0001\right)$ (Fig. 8). 


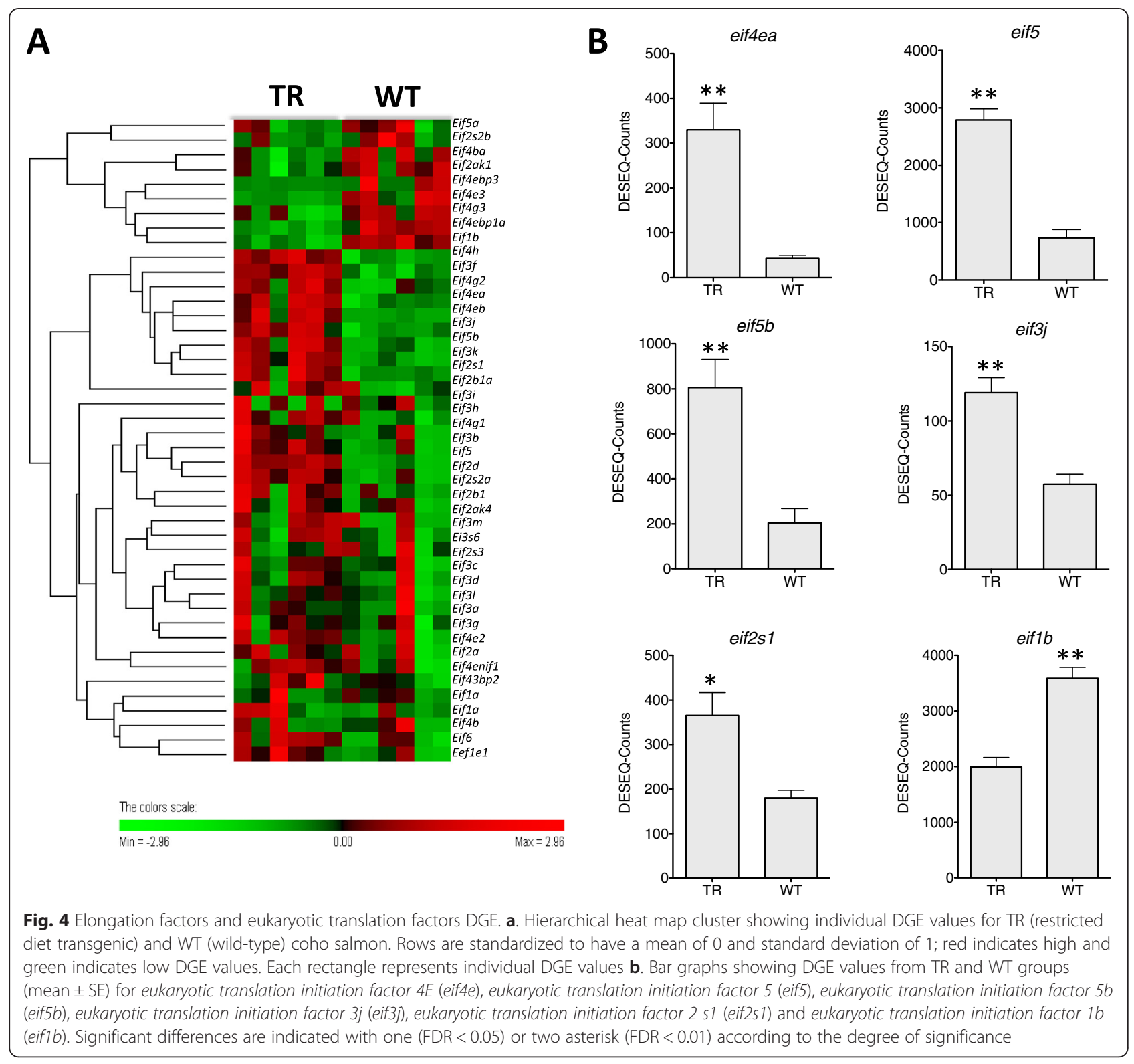

Energy budgeting in the restriction-fed transgenic model TR fish are more aggressive and exhibit markedly higher levels routine swimming behaviour than WT fish and may have higher metabolic costs associated with the futile expression of mRNAs and proteins required for growth (present study). In spite of these additional costs, TR fish grow at the same rate as WT fish which implies some compensating alterations to energy budgeting. The source of these energy savings includes the cost of ionic homeostasis which has been estimate to contribute $20-40 \%$ of the routine metabolic rate in teleosts [15], a large part of which can be attributable to maintaining the resting membrane potential of fast skeletal muscle which comprises around $70 \%$ of body mass in salmonids. TR fish have fewer, but larger diameter fast muscle fibres than WT for a given body length [13], which will result in reduced ion pumping costs [14, 15]. Our working hypothesis is that the uncoupling of the Ghaxis from energy status directly affects one or more of the signaling pathways regulating myotube formation and hypertrophic growth, and several candidate pathways, particularly signaling through cadherin, have been identified in the present study. Calorie restriction in WT fish may not result in changes in muscle cellularity because the metabolic signals normally influencing endogenous $G$ h regulation remain intact, a hypothesis requiring further assessment. 


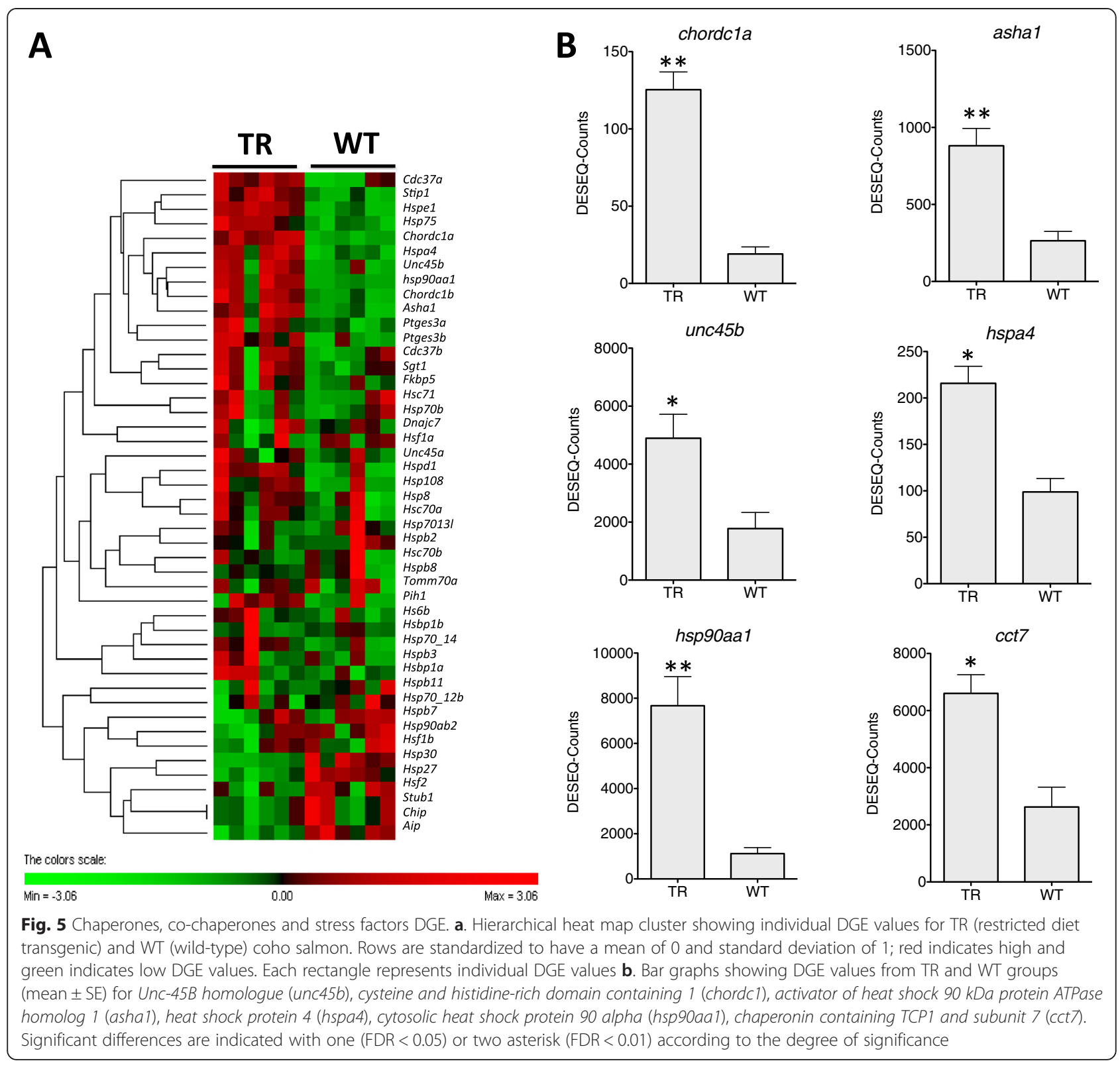

\section{Methods}

Fish

Experiments on coho salmon (Oncorhynchus kisutch) were conducted in a non-commercial containment facility for transgenic fish at Fisheries and Oceans Canada, West Vancouver. Wild-type (WT) fish were from the 2010 brood of Chehalis River strain (British Columbia, Canada) [33]. The strain of transgenic coho salmon used (M77) was derived from Chehalis River strain produced using the OnMTGH1 construct comprised of $320 \mathrm{bp}$ of sockeye salmon metallothionein- $\beta$ promoter fused to the 5 '-UTR region of the full-length type-I growth hormone (gh1) gene and the terminator from the same species, as previously described [33]. Fish were reared under standard hatchery conditions in fresh well water $\left(10 \pm 1{ }^{\circ} \mathrm{C}\right)$ with a natural photoperiod and fed commercially available salmon diets (Skretting, Vancouver, Canada). The 2010 brood of Gh transgenics (TR group) were fed the same ration to that of the WT group (i.e. pair fed), resulting in similar growth rates in the two groups. Fish were fasted for $24 \mathrm{~h}$ prior to humane sacrifice and selected to produce two groups with a similar average fork length $(\mathrm{cm})$ and hence same the developmental stage. All procedures used in the study were approved by the Department of Fisheries and Oceans Pacific Region Animal Care Committee. WT and food-restricted growth hormone transgenic coho salmon (TR) were obtained from the same group used by the authors in a previous study [13]. No significant differences 


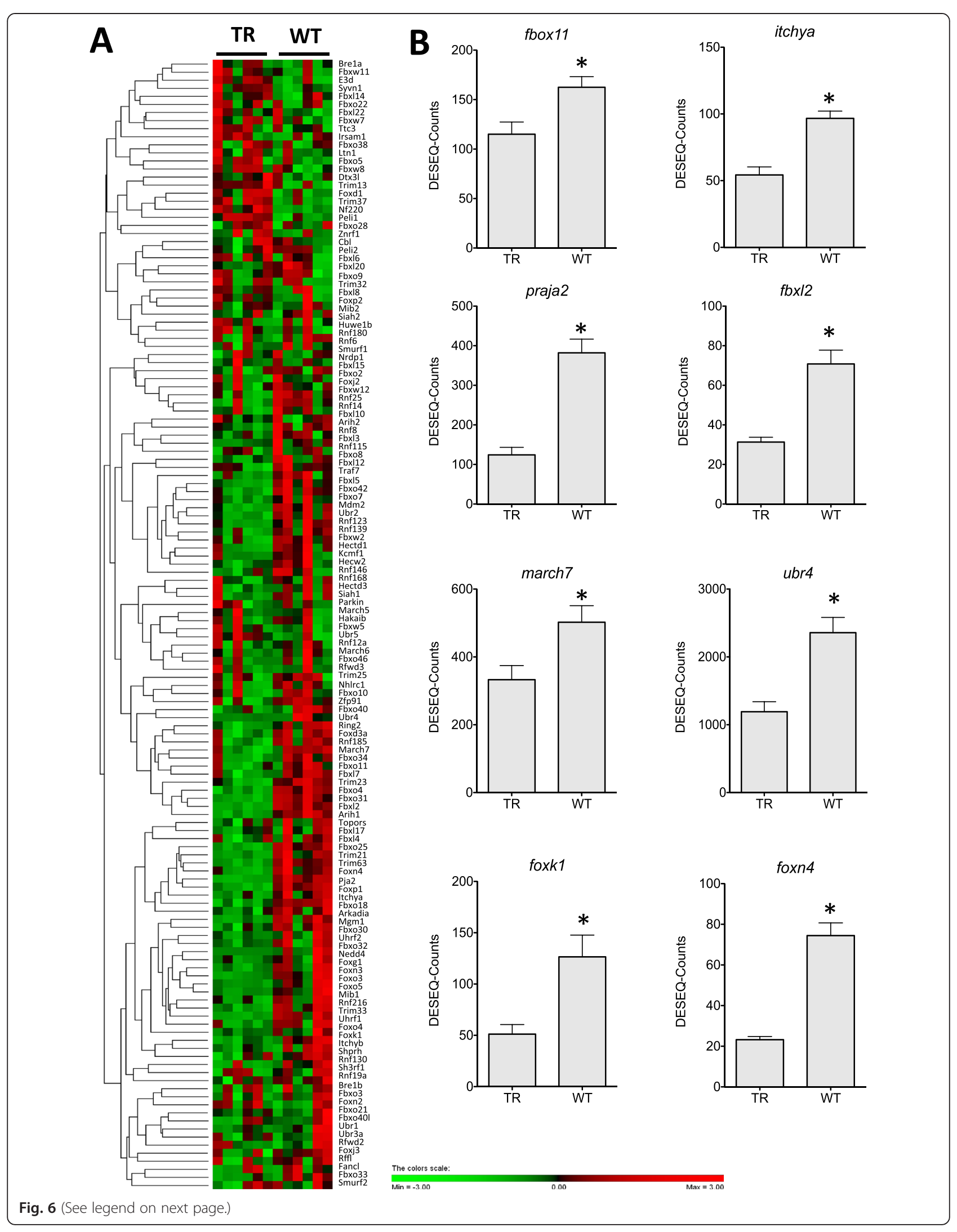


(See figure on previous page.)

Fig. 6 E3-ubiquitin ligases DGE. a. Hierarchical heat map cluster showing individual DGE values for TR (restricted diet transgenic) and WT (wild-type) coho salmon. Rows are standardized to have a mean of 0 and standard deviation of 1 ; red indicates high and green indicates Iow DGE values. Each rectangle represents individual DGE values $\mathbf{b}$. Bar graphs showing DGE values from TR and WT groups (mean \pm SE) for F-box protein 11 (fbox 11), E3-ubiquitin ligase Itchya (itchya), F-box and leucine-rich repeat protein 2 (fbx/2), praja ring finger 2 E3 ubiquitin protein ligase (praja2), ubiquitin protein ligase E3 component n-recognin 4 (ubr4), membrane-associated ring finger (C3HC4) 7 E3 ubiquitin protein ligase (march7), forkhead box K1 (foxk1) and forkhead box N4 (foxn4). Significant differences are indicated with one (FDR < 0.05) or two asterisk $($ FDR $<0.01)$ according to the degree of significance

were found in weight or fork length between TR $(63.5 \pm$ $3.1 \mathrm{~g} ; 17.4 \pm 0.2 \mathrm{~cm})$ and WT $(57.7 \pm 2 \mathrm{~g} ; 17.1 \pm 0.1 \mathrm{~cm})$ used in the present study.

\section{RNA extraction and sequencing}

Sections of pure fast skeletal muscle were carefully dissected from dorsal epaxial myotomes of 6 animals from WT and TR groups matched for body length and with known differences in muscle fibre distribution [13]. Total RNA was extracted by homogenization in $1 \mathrm{ml}$ of Trisure (Bioline, London, UK) using D-Matrix tubes (MP Biomedical, Cambridge, UK) and following the manufacturer's recommendations. Total RNA concentration, 260/230 and 260/280 ratios were estimated by Nanodrop spectrometer N1000 (Thermo Scientific). RNA integrity was estimated by resolving $1 \mu \mathrm{g}$ of sample in a $1 \%(\mathrm{~m} / \mathrm{v})$ ethidium bromide agarose gel. A total of $3 \mu \mathrm{g}$ of RNA per sample was sent to the Sick-Kids Hospital Next Generation Sequencing service (Vancouver, Canada) for sequencing. Individual barcoded libraries for each animal were paired-end sequenced using two lines of Illumina HiSeq2000. Raw reads were deposited in the EBISRA database under the accession number PRJEB7712.

\section{CDNA synthesis and qPCR reactions}

$1 \mu \mathrm{g}$ of total RNA from 6 individuals for each of the treatments (WT and TR) was reverse transcribed to cDNA using the Quantitec reverse transcription kit (QIAGEN, Manchester, UK) including the gDNA wipeout step to remove remains of genomic DNA and RT and NT controls were run in parallel with $1 \mu \mathrm{g}$ of RNA but no RT enzyme or RT enzyme but no template. cDNA samples were diluted $1 / 50$ in Nuclease-free water (QIAGEN). $6 \mu \mathrm{l}$ per sample were mixed with $7.5 \mu \mathrm{l}$ of SensiFAST SYBR Lo-ROX 2X master mix (Bioline) containing $400 \mathrm{nM}$ of sense/antisense primers. Reactions were performed in duplicated in a Mx3005P thermocycler (Agilent, Oxford, UK), with 1 cycle of $2 \mathrm{~min}$ at $95^{\circ} \mathrm{C}$ and $\mathrm{x} 40$ cycles of $5 \mathrm{~s} 95^{\circ} \mathrm{C}$ and $20 \mathrm{~s}$ at $65^{\circ} \mathrm{C}$, followed by a dissociation curve analysis, where a single peak was detected in all cases.

Primers were designed using Primer 3 [http://biotools.umassmed.edu/bioapps/primer3_www.cgi] to amplify products between 100-200 bp from gene sequences retrieved from a de novo coho salmon skeletal muscle transcriptome (primers and sequences used for primer design can be found in Additional file 11). Netprimer (PremierBiosoft) [http://www.premierbiosoft.com/netprimer/] was used to detect primer hairpins and cross-dimmers. When two salmonid paralogues were identified primers were design to bind to the most divergent regions of sequence. Genorm software [34] was used to evaluate the stability of the four reference genes analysed rpl27, rpl13, efla and ßactin. The rpl13 and efla were found to be the most stable reference genes $(M=0.058)$. Normalization of gene expression was performed using the geometric average of rpl13 and efla. All expression values are expressed as arbitrary units.

\section{De novo transcriptome assembly and annotation}

Quality filtered raw reads were de novo assembled by SickKids Bioinformatic services (Vancouver, Canada) using Trinity software [35] (the complete de novo assembly can be found in the Additional files 12, 13, 14, 15 and 16). Resulting contigs were identified by BLAST (BLASTx) against the NCBI non-redundant database (nr) and Gene Ontology (GO) annotated using Blast2GO software default settings [36]. In order to identify the number of unique genes BLASTx results were manually investigated to remove duplicates and those contigs annotated as hypothetical proteins or predicted proteins.

Fully annotated contigs (with positive BLAST and GO results) were BLAST against the KEGG collection of metabolic and signaling pathways with the KEGG Automatic Annotation Service (KAAS) using the single-directional best hit $(\mathrm{SBH})$ method against all vertebrates pathways deposited in the database [37]. For identification of coding sequences in the contigs generated during the de novo assembly the complete zebrafish proteome [38] was blasted (tBLASTn) against all annotated contigs using BioEdit software [www.mbio.ncsu.edu/bioedit/bioedit.html]. Alignment data from positive hits results between coho salmon contigs and zebrafish gene amino acid sequences gave us an estimation of the percentage of coding sequences contained in the de novo contigs.

\section{Digital gene expression}

Read mapping, read normalization and global digital expression were carried out by the bioinformatics department 
A
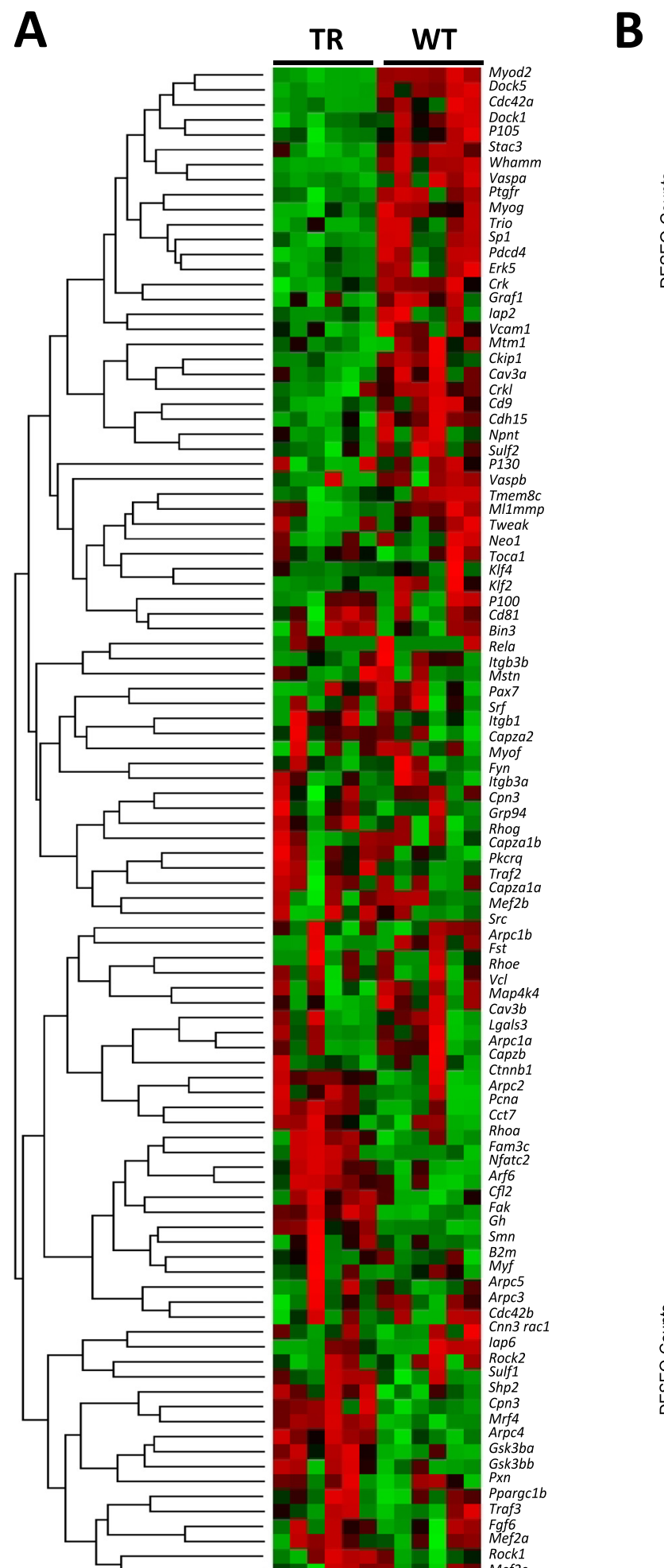

The colors scale:

pargc1b

$M a n=311$
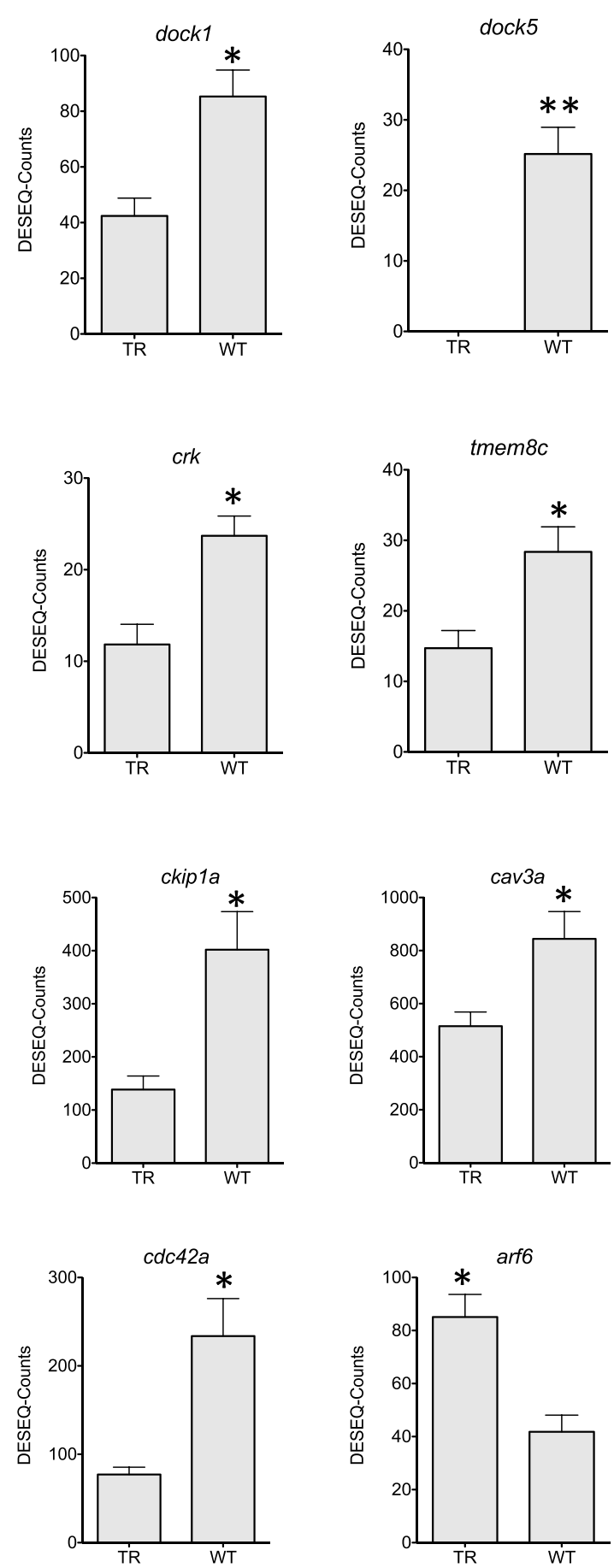

政 
(See figure on previous page.)

Fig. 7 Myoblast fusion related genes DGE. a. Hierarchical heat map cluster showing individual DGE values for TR (restricted diet transgenic) and WT (wild-type) coho salmon. Rows are standardized to have a mean of 0 and standard deviation of 1 ; red indicates high and green indicates low DGE values. Each rectangle represents individual DGE values $\mathbf{b}$. Bar graphs showing DGE values from TR and WT groups (mean \pm SE) for dedicator of cytokinesis 1 (dock1), dedicator of cytokinesis 5 (dock5), v-crk avian sarcoma virus CT10 oncogene homolog (crk), transmembrane protein 8C (tmem8c), pleckstrin homology domain containing, family 0 member 1 (ckip 1a), caveolin $3 a$ (cav3a). Significant differences are indicated with one (FDR $<0.05)$ or two asterisk (FDR $<0.01)$ according to the degree of significance

of Sick-Kids Hospital, Next Generation Sequencing service (Vancouver, Canada). Quality filtered raw reads from individual libraries were mapped to the complete de novo coho salmon transcriptome and their abundance estimated using the RSEM [39]. DESEQ program from the R-Bioconductor package was used to estimate global differences in digital gene expression (DGE) between TR and WT groups [40]. The DESEQ program normalizes mapped reads for individual samples by contig length and library depth using a negative binomial distribution previous to test differences in reads mapped between conditions. To test specific hypothesis for particular physiological processes DESEQ normalized counts (DESEQ-counts) per individual animal were retrieved.

\section{Gene ontology (GO) enrichment analysis}

GO enrichment analysis from those genes differently expressed between TR and WT after the global DGE analysis was performed using the STRING webserver [41]. In order to maximize GO enrichment analysis BLAST results were manually annotated with their human orthologue abbreviated gene name (e.g. contig identified as dedicator of cytokinesis protein 5 was named as DOCK5).

\section{Paralogue identification and phylogenetic analysis}

A phylogenetic analysis was carried to confirm the salmonid-specific WGD origin of potential $4 \mathrm{R}$ paralogues in the transcriptome. Potential 4R-paralogues were conceptually translated to their amino acid sequence. BLASTp against the non-redundant NCBI database was used to confirm the identities of the translated paralogues. Teleost orthologues for the genes of interest were retrieved from Ensembl including human and mouse orthologues to be used as outgroups. Potential coho salmon $4 \mathrm{R}$ paralogues were used as a query against the rainbow trout (Oncorhynchus mykiss) protein collection (BLASTp) and the Atlantic salmon (Salmo salar) Transcriptome Shotgun Assembly (TSA) data deposited in the NCBI (tBLASTn). Positive BLAST hits were included in the analysis. Amino acids sequences were aligned using Guidance webserver [42] with MAFFT as MSA algorithm. Only aligned sections with a score over 0.93 were used to generate the phylogenetic tree. Evolutionary models were estimated for all alignments using MEGA5 [43]. In all cases the best evolutionary model was estimated to be JTT + G (data not show). Finally Maximum likelihood trees for each alignment were constructed using PhyML webserver [44] and displayed using FigTree [http://tree.bio.ed.ac.uk/software/figtree].
A

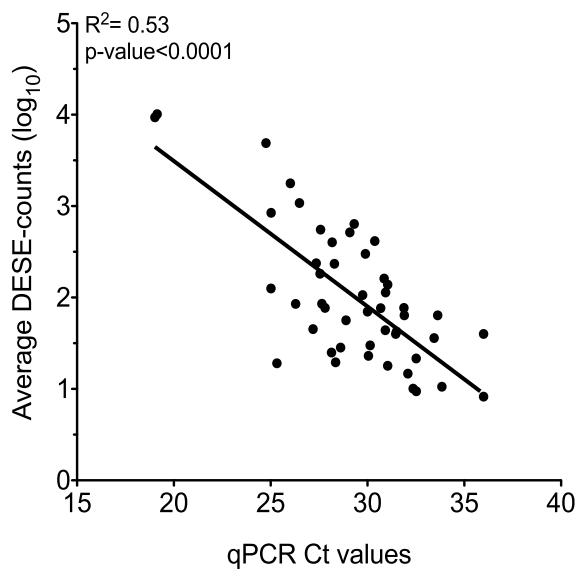

\section{B}

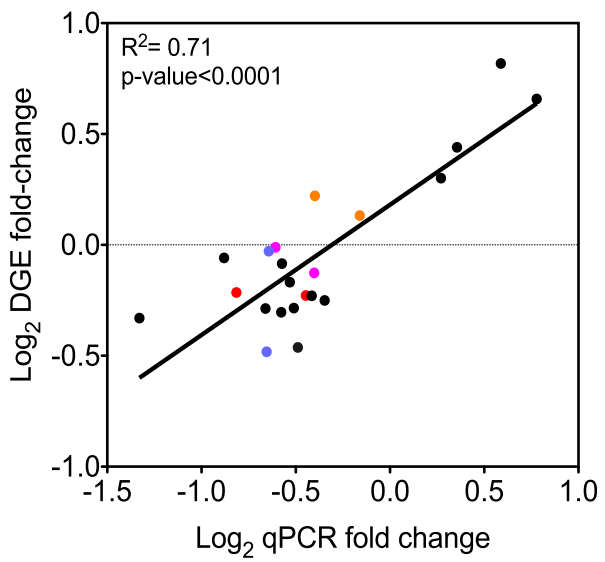

Fig. 8 DGE and qPCR correlations. a. DGE and qPCR correlations. a. Correlation between DGE and qPCR Ct values for individual genes for each treatment. DGE values are expressed as $\log _{10}$, Pearson $R^{2}$ and P-value for the correlation are indicated in the top-left corner b. Correlation between

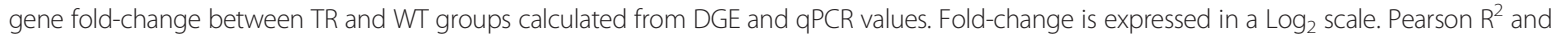
P-values for the correlation are indicated in the top-left corner. Circles with different colour than black represent the position of the 4R-paralogues plotted: cav3a/cav3b (red circles), cdc42a/cdc42b (blue circles), ghra.2/ghrb.1 (pink circles) and vaspa/vaspb (orange circles) 


\section{Statistical analysis}

For global DGE between TR and WT individuals the DESEQ program was used following programmer recommendations and a false discovery rate (FDR) cut-off of FDR $<0.05$ was applied for significant differences. The original list of genes differently expressed generated by DESEQ was manually curated and only those contigs with >15 DESEQ-counts and over $2 \log _{2}$ fold-change were used. As expected from previous studies [45, 46], coho salmon de novo transcriptome presented a significant degree of redundancy; therefore, more than one contig shared the same BLAST results ("sister" contigs). The original DESEQ list of genes was further curated, maintaining those genes that showed consistent expression between "sister contigs", special care was taken to detect $3 \mathrm{R}$ and 4R-paralogues annotated with the same ID by investigating the alignment of sister contigs.

Differences in expression for specific groups of genes were estimated using DESEQ-counts values per individual animal for each gene $(n=6$ for each experimental group). Significant differences were detected by a two-tailed test between WT and TR individuals followed by a Benjamin-Hochberg (FDR) correction for multiple comparisons. Significant differences were accepted when FDR $<0.05$.

Hierarchical clustering analysis of gene expression data was performed using Permutmatrix with gene expression normalized by row using McQuitty's method [47]. Principal component analysis for gene expression of the 12 coho salmon analysed was performed using PASW Statistic software v21 (IBM).

Differences in gene expression analysed by qPCR were analysed by t-test and a significant threshold of $P<0.05$. Pearson correlation was used to study qPCR and DGE expression data obtained for the same genes. In order to facilitate visualization of results data was logarithmically transformed $\left(\log _{10}\right.$ for expression data and $\log _{2}$ for fold-change data) prior to correlation analysis. Pearson correlation was estimated using PASW Statistic software v21.

\section{Conclusions}

Gh-transgenic coho salmon show increased appetite and growth relative to wild type fish. We restricted the food intake of transgenic fish (TR) to that of wild-type (WT) fish fed to satiation, resulting in higher levels of muscle Gh expression, but a similar final body size. The two groups had markedly different gene expression signatures, with TR fish showing increased transcript abundance for pathways associated with protein translation and protein folding and reduced expression of genes involved with myotube fusion. The down-regulation of genes with known function in myoblast fusion, particularly cadherin signaling, was correlated with a reduction in average muscle fibre diameter in TR relative to WT fish which is expected to reduce the costs of maintaining ionic homeostasis. This may explain why TR fish are more active than WT fish yet grow at a similar rate.

\section{Availability of supporting data}

All supporting data for the present manuscript is included as additional files.

\section{Additional files}

\begin{abstract}
Additional file 1: Annotated transcriptome sequences (.zip), sequences were annotated using BLAS2GO (BLASTx) against the $\mathrm{NCBI}$ non-redundant database (nr) unigene IDs present in the transcriptome (.txt). KAAS file containing mapping results (.hier) and the transcriptome was annotated against all vertebrate pathways present in the KAAS online server. (ZIP $13705 \mathrm{~kb}$ )

Additional file 2: Gene sequences containing $>90 \%$ of the coding sequence (.fasta). Coding sequence coverage distribution from annotated unigenes present in the coho salmon transcriptome (shaded area represents those contigs containing 100 to $50 \%$ of the coding sequence). (PDF $316 \mathrm{~kb}$ )

Additional file 3: Paired-end reads generated for each individual library and the percentage of assembly. (XLSX $54 \mathrm{~kb}$ )
\end{abstract}

Additional file 4: Genes with significant changes in their expression between restriction-fed growth hormone-transgenic coho salmon (TR) and wild-type salmon (WT) after DESEQ analysis of the transcriptome as a whole (.excel). (XLSX $61 \mathrm{~kb}$ )

Additional file 5: Maximum-likelihood phylogenetic trees, genes sequences and alignments used to resolve $4 \mathrm{R}$-salmonid paralogues found during the study. (ZIP $4 \mathrm{~kb}$ )

Additional file 6: Genes related to myoblast fusion, the Gh-lgf network, chaperones/co-chaperones, initiation factors, E3-ubiquitin ligases, initiation factors and autophagy, retrieved from the transcriptome to test specific hypothesis. WT $1-5$ and TR $1-5$ values represent DESEQ-counts for individual animals. (XLSX $171 \mathrm{~kb}$ )

Additional file 7: DGE results of genes involved with initiation of protein translation. (DOCX $102 \mathrm{~kb}$ )

Additional file 8: DGE results of genes involved with protein folding. (DOCX $79 \mathrm{~kb}$ )

Additional file 9: DGE results of genes involved with myoblast fusion. (DOCX $105 \mathrm{~kb}$ )

Additional file 10: Bar graphs of qPCR results for individual genes. Gene expression is expressed in arbitrary units (a.u). Significant differences between groups are indicated with an asterisk $(P<0.05)$. (PDF $402 \mathrm{~kb}$ )

Additional file 11: Quantitative PCR primer sequences, PCR efficiencies and melting temperatures for genes analysed (DOCX $143 \mathrm{~kb}$ )

Additional file 12: de novo coho salmon transcriptome (part 1 of 5). (ZIP $14691 \mathrm{~kb}$ )

Additional file 13: de novo coho salmon transcriptome (part 2 of 5). (ZIP $10798 \mathrm{~kb}$ )

Additional file 14: de novo coho salmon transcriptome (part 3 of 5). (ZIP 9435 kb)

Additional file 15: de novo coho salmon transcriptome (part 4 of 5). (ZIP $5145 \mathrm{~kb}$ )

Additional file 16: de novo coho salmon transcriptome (part 5 of 5). (ZIP 8207 kb)

Competing interests

The authors declare that they have no competing interests. 


\section{Authors' contributions}

DGS carried out the bioinformatic and digital gene expression analysis with support of IAJ. DGS performed the statistical analysis. RHD was responsible of the fish husbandry, generation of the experimental groups and provided the samples. DGS and IAJ wrote the manuscript with RHD inputs. IAJ and RHD conceived the study. All authors read and approved the final manuscript.

\section{Acknowledgements}

The study was supported by the Marine Alliance for Science and Technology for Scotland (Scottish Funding Council grant HR09011) and by the Canadian Biotechnology Strategy (RHD).

\section{Author details}

${ }^{1}$ Scottish Oceans Institute, School of Biology, University of St Andrews, KY16 8 LB St Andrews, Scotland, UK. ²Department of Fisheries and Oceans, Centre for Aquaculture and Environmental Research, 4160 Marine Drive, West Vancouver, BC V7V 1N6, Canada.

Received: 31 March 2015 Accepted: 15 July 2015

Published online: 31 July 2015

\section{References}

1. Bjornsson BT, Johanson V, Benedet S, Einarsdottir IE, Hildahl J, Agustsson T, et al. Growth hormone endocrinology of salmonids: regulatory mechanism and mode of action. Fish Physiol Biochem. 2002;27:227-42.

2. Mori T, Devlin RH. Transgene and host growth hormone gene expression in pituitary and nonpituitary tissues of normal and growth hormone transgenic salmon. Mol Cel Endocrinol. 1999;149:129-39.

3. Raven PA, Sakhrani MU, Beckman BR, Cooper K, Pinter J, Leder EH. Endocrine effects of growth hormone overexpression in transgenic coho salmon. Gen Comp Endocrinol. 2008;159:26-37.

4. Devlin BH, Timothy TT, Edward MD, Choy-Leong H. Transmission and phenotypic effect of an antifreeze/GH gene construct in coho salmon (Oncorhynchus kisutch). Aquaculture. 1995;137:161-9.

5. Devlin BH, D'Andrade M, Uh M, Biagi CA. Population effects of growth hormone transgenic coho salmon depend on food availability and genotype by environment interactions. Proc Natl Acad Sci U S A. 2004;101:9303-8.

6. Devlin RH, Sakhrani D, Tymchuk WE, Rise ML, Goh B. Domestication and growth hormone transgenesis cause similar changes in gene expression in coho salmon (Oncorhynchus kisutch). Proc Natl Acad Sci U S A. 2009;106:3047-52.

7. Bower $\mathrm{NI}$, Johnston IA. Transcriptional regulation of the IGF signaling pathway by amino acids and insulin-like growth factors during myogenesis in Atlantic salmon. PLOS ONE. 2010:42, e11100.

8. Garcia de la serrana D, Johnston IA. Expression of heat shock protein 90 (Hsp90) paralogues is regulated by amino acids in skeletal muscle of Atlantic salmon. PLoS ONE. 2013;8:e74295.

9. Rowlerson A, Mascarello F, Radaelli G, Veggetti A. Differentiation and growth of muscle in the fish Sparus aurata $(\mathrm{L})$ : II. Hyperplastic and hypertrophic growth of lateral muscle from hatching to adult. J Mus Res Cell Mot. 1995:3:223-36.

10. Johnston IA, Cole NJ, Abercromby M, Vieira VLA. Embryonic temperature modulates muscle growth characteristics in larval and juvenile herring. J Exp Biol. 1998;201:623-46.

11. Weatherley AH, Gill HS, Lobo AF. Recruitment and maximum diameter of axial muscle fibres in teleost and their relationship to somatic growth and ultimate size. J Fish Biol. 1988:33:851-9.

12. Hill JA, Kiessling A, Devlin RH. Coho salmon (Oncorhynchus kisutch) transgenic for growth hormone gene construct exhibit increased rates of hyperplasia and detectable levels of differential gene expression. Can J Aquat Sci. 2000:57:939-50.

13. Johnston IA, Garcia de la serrana D, Devlin RH. Muscle fibre size optimisation provides flexibility to energy budgeting in caloric-restricted Coho salmon transgenic for growth hormone. J Exp Biol. 2014;217:3392-95. doi:10.1242/jeb.107664.

14. Johnston IA, Kristjánsson BK, Paxton CG, Vieira VLA, Macqueen DJ, Bell MA Universal scaling rules predict evolutionary patterns of myogenesis is species with indeterminate growth. Proc R Sco B. 2012:279:2255-61.

15. Jimenez AG, Dillaman RM, Kinsey ST. Large fibre size in skeletal muscle is metabolically advantageous. Nature Comm. 2013;4:2150. doi:10.1038/ncomms3150.
16. Glasauer SMK, Neuhauss SCF. Whole-genome duplication in teleost fishes and its evolutionary consequences. Mol Gen Genom. 2014;289:1045-60.

17. Garcia de la serrana D, Mareco EA, Johnston IA. Systematic variation in the pattern of gene paralog retention between the teleost superorders Ostariophysi and Acanthopterygii. Gen Biol Evol. 2014;6:981-7.

18. Macqueen DJ, Johnston IA. A well-constrained estimate for the timing of the salmonid whole genome duplication reveals major decoupling from species diversification. Proc R Soc B Biol Scie. 2014;281:20132881.

19. Macqueen DJ, Johnston IA. Evolution of the multifaceted eukaryotic akirin gene family. BMC Evol Biol. 2009;9:34

20. Macqueen DJ, Garcia de la serrana D, Johnston IA. Evolution of ancient functions in the vertebrate insulin-like growth factor system uncovered by study of duplicated salmonid fish genomes. Mol Biol Evol. 2013;30:1060-76.

21. Lindholm ME, Huss M, Solnestam BW, Kjellqvist S, Lunderberg J, Sundberg C. The human skeletal muscle transcriptome: sex differences, alternative splicing, and tissue homogeneity assessed with RNA sequencing. FASEB J. 2014:28:4571-81.

22. Choo AY, Yoon SO, Kim SG, Roux PP, Blenis J. Rapamycin differentially inhibits S6Ks and E4-BP1 to mediate cell-type-specific repression of mRNA translation. Proc Natl Acad Sci. 2008;105:17414-9.

23. Gringas AC, Raught B, Gygi SP, Niedzwiecka A, Miron M, Burley SK, et al. Hierarchical phosphorylation of the translation inhibitor 4E-BP1. Gen Dev. 2001:15:2852-64

24. Huang K, Fingar DC. Growing knowledge of the mTOR signaling network. Sem Cell Dev Biol. 2014;36:79-90.

25. Sancak Y, Bar-Peled L, Zoncu R, Markhard AL, Nada S, Sabatini DM Ragulator-Rag compex target mTORC1 to the lysosomal surface and is necessary for its activation by amino acids. Cell. 2010;141:290-303.

26. Roccio M, Bos JL, Zwarkruis FJT. Regulation of the small GTPase Rheb by amino acids. Oncogene. 2006:25:657-64.

27. Du J, Bernick E. Molecular chaperones Hsp90a1 and Unc45b are required for sarcomere assembly in skeletal muscles of zebrafish embryos. FASEB. 2011;28:10.

28. Hindi SM, Marjan M, Kumar A. Signaling Mechanisms in Mammalian Myoblast Fusion. Sci Signal. 2013;6:272.

29. Moore CA, Parkin CA, Bidet $Y$, Ingham PW. A role for the Myoblast city homologues Dock1 and Dock5 and the adaptors Crk and Crk-like in zebrafish myoblast fusion. Develop. 2007;134:3145-53.

30. Charrasse S, Comunale F, Grumbach Y, Poulat F, Blangy A, Gauthier-Rouviére C. RhoA GTPase regulates $\mathrm{m}$-cahderin activity and myoblast fusion. Mol Biol Cell. 2006;17:749-59.

31. Millay DP, O'Rourke JR, Sutherland LB, Bezprozvannya S, Shelton JM, Bassel-Duby R, et al. Myomaker is a membrane activator of myoblast fusion and muscle formation. Nature. 2013;499:301-5.

32. Quach NL, Biressi S, Reichardt LF, Keller C, Rando TA. Focal adhesion kinase signalling regulates the expression of caveolin 3 and beta integrin, genes essential for normal myoblast fusion. Mol Biol Cell. 2009;14:3422-55.

33. Devlin RH, Biagi CA, Yesaki TY. Growth, viability and genetic characteristics of $\mathrm{GH}$ transgenic coho salmon strains. Aquaculture. 2004;236:607-32.

34. Vandesompele J, De Preter K, Pattyn F, Poppe B, Van Roy N, De Paepe A, et al. Accurate normalization of real-time quantitative RT-PCR data by geometric averaging of multiple internal control genes. Genom Biol. 2002;3:research0034research0034.11.

35. Hass BJ, Papanicolau A, Yassour M, Grabherr M, Blood PD, Bowden, et al. De novo transcript sequence reconstruction from RNA-seq: reference generation and analysis with Trinity. Nat Protoc. 2013;8:1494-512.

36. Götz S, García-Gómez JM, Terol J, Williams TD, Nagaraj SH, Nueda MJ et al. High-throughput functional annotation and data mining with the Blast2GO suite. Nucl Acids. 2008;36:3420-35

37. Moriya I, Itoh M, Okuda S, Yoshizama A, Kanehisa M. KAAS: an automatic genome annotation and pathway reconstruction server. Nucl Acids. 2007:35:182-5

38. www.ensembl.org (last entrance March 2014).

39. Li B, Dewey CN. RSEM: accurate transcript quantification from RNA-seq data with or without a reference genome. BMC Bioinformatics. 2011:12:323.

40. Anders $\mathrm{S}$, Huber W. Differential expression analysis of sequence count data. Genom Biol. 2010:11:106.

41. Franceschini A, Szklarczyk D, Frankild S, Kuhn M, Simonovic M, Roth A, et al. STRING v9.1: protein-protein interaction networks, with increase coverage and integration. Nucl Acids. 2013;41:808-15. 
42. Penn O, Privman E, Ashkenazy H, Landan G, Graur D, Pupko T. GUIDANCE: a web server for assessing alignment confidence scores. Nucl Acids Res. 2010;1:W23-8.

43. Tamura K, Stecher G, Peterson D, Filipski A, Kumar S. MEGA6: Molecular Evolutionary Genetics Analysis Version 6.0. Mol Biol Evol. 2013;30:2725-9.

44. Dereeper A, Guignon V, Blanc G, Audic S, Buffet S, Chevenet F, et al. Phylogeny.fr: robust phylogenetic analysis for the non-specialist. Nucl Acids Res. 2008:36:465-9.

45. Garcia de la serrana D, Estevez A, Andree K, Johnston IA. Fast skeletal muscle transcriptome of the Gilthead sea bream (Sparus aurata) determined by next generation sequencing. BMC Genomics. 2012;13:181.

46. Mareco EA, Garcia de la serrana D, Johnston IA, Dal-Pai-Silva M. Characterization of the transcriptome of fast and slow muscle myotomal fibres in the pacu (Piaractus mesopotamicus). BMC Genomics. 2015. doi:10.1186/s12864-015-1423-6.

47. Caraux G, Pinloche S, Permutmatrix. A graphical environment to arrange gene expression profiles in optimal linear order. Bioinformatics. 2005;21:1280-1.

\section{Submit your next manuscript to BioMed Central and take full advantage of:}

- Convenient online submission

- Thorough peer review

- No space constraints or color figure charges

- Immediate publication on acceptance

- Inclusion in PubMed, CAS, Scopus and Google Scholar

- Research which is freely available for redistribution 